IBT-23

\title{
High Intensity, Pulsed, D-D Neutron Generator
}

\author{
D. L. Williams, J. H. Vainionpaa, G. Jones, M. A. Piestrup, C. K. Gary, J. L. Harris, M. J. Fuller, J. T. \\ Cremer \\ Adelphi Technology, Inc., 2003 E Bayshore Rd, Redwood City, CA 94063
}

B. A. Ludewigt, J. W. Kwan, J. Reijonen ', K.-N. Leung, R. A. Gough

Lawrence Berkeley National Laboratory, 1 Cyclotron Rd. Berkeley CA 94720

\section{ACKNOWLEDGMENT}

This work was supported by the US Department of Homeland Security under Contract no. HSHQDC-08C-00022 and the US Department of Energy under contract no. DE-FG02-04ER86177, and DE-AC02$05 \mathrm{CH} 11231$.

\section{DISCLAIMER}

This document was prepared as an account of work sponsored by the United States Government. While this document is believed to contain correct information, neither the United States Government nor any agency. thereof, nor The Regents of the University of California, nor any of their employees, makes any warranty, express or implied, or assumes any legal responsibility for the accuracy, completeness, or usefulness of any information, apparatus, product, or process disclosed, or represents that its use would not infringe privately owned rights. Reference herein to any specific commercial product, process, or service by its trade name, trademark, manufacturer, or otherwise, does not necessarily constitute or imply its endorsement, recommendation, or favoring by the United States Government or any agency thereof, or The Regents of the University of California. The views and opinions of authors expressed herein do not necessarily state or reflect those of the United States Government or any agency thereof or The Regents of the University of California. 
Manuscript ID: 525

2 Title: High Intensity, Pulsed, D-D Neutron Generator

3 Authors: D. L. Williams, J. H. Vainionpaa, G. Jones, M. A. Piestrup, C. K. Gary, J. L. Harris, M. J. Fuller, J. T.

4 Cremer

5 Affiliation: Adelphi Technology, Inc., 2003 E Bayshore Rd, Redwood City, CA 94063

6 Authors: B. A. Ludewigt, J. W. Kwan, J. Reijonen ${ }^{1}$, K.-N. Leung, R. A. Gough

7 Affiliation: Lawrence Berkeley National Laboratory, 1 Cyclotron Rd. Berkeley CA 94720

9 Abstract:

10 Single ion-beam RF-plasma neutron generators are presented as a laboratory source of intense neutrons. The 11 continuous and pulsed operations of such a neutron generator using the deuterium-deuterium fusion reaction are 12 reported. The neutron beam can be pulsed by switching the RF plasma and/or a gate electrode. These generators are 13 actively vacuum pumped so that a continuous supply of deuterium gas is present for the production of ions and

14 neutrons. This contributes to the generator's long life. These single-beam generators are capable of producing up to $1510^{10} \mathrm{n} / \mathrm{s}$. Previously, Adelphi and LBNL have demonstrated these generators' applications in fast neutron 16 radiography, Prompt Gamma Neutron Activation Analysis (PGNAA) and Neutron Activation Analysis (NAA).

17 Together with an inexpensive compact moderator, these high-output neutron generators extend useful applications to 18 home laboratory operations.

20 Keywords: neutron generator, prompt gamma activation analysis, neutron spectroscopy, neutron source.

21 PACS codes:29.25.Dz, 28.20.-v, 14.20.Dh

\section{Plasma Neutron Generators}

24 RFI plasma neutron generators can produce intense neutron outputs of $10^{10} \mathrm{n} / \mathrm{s}$ or greater with the deuterium25 deuterium (D-D) reaction [1]. Based on core technology originally developed at Lawrence Berkeley National 26 Laboratory [1-3] and further developed by Adelphi, these generators provide many times greater neutron output than 27 other portable/transportable systems and have proven to be reliable and easily serviced.

\footnotetext{
${ }^{1}$ Now at Schlumberger
} 
As shown in Fig. 1, plasma ion source neutron generators are either simple diodes or triodes with an RF ion plasma source. This source generates the $\mathrm{D}^{+}$ions that are then accelerated across a potential of $\sim 120 \mathrm{kV}$ to a titanium target. In the triode, the ion beam can be gated as is the case in Fig. 1. The target is biased to a negative voltage, while the plasma is at ground. The negative potential will then 'attract' the positively charged particles to the target. The accelerated ions impinge onto a titanium coated copper target where 2.5-MeV D-D neutrons are generated through fusion reactions. The implanted deuterium ions form titanium hydrates in the titanium matrix, thus 'trapping' the deuterium atoms. When the generator is operating, the titanium layer is initially being loaded with deuterium and the neutron output is increasing with time; then after some minutes of operation the target is

9 loaded by the incoming ions and the neutron output saturates to a stable level. While the ions are implanted to the target, heat is also deposited, which is removed from the target by water-cooling it.

Advantages of using the RF-induction method compared to other types of discharge, like the penning and

12 filament discharge, are (1) its ability to generate high plasma densities for a high intensity ion beam production, (2)

13 its ability to generate a high percentage of atomic ion species, and (3) its long lifetime operation. A high atomic ion

14 species fraction is important for the overall efficiency of the neutron generator. Neutron output increases by a factor 15 of 3-4 if one uses a $100 \%$ atomic ion beam in comparison to a $100 \%$ molecular ion beam. The RF-induction 16 discharge generates more than 95\% of atomic ion species at about $2 \mathrm{~kW}$ of discharge power [1-4].

17 These generators use an active pumping method wherein the deuterium is continually replenished to maintain 18 continuous operation with no loss of neutron yield over many thousands of hours of operation. Other generators 19 currently available on the market use radioactive tritium gas, are sealed, and have a lifetime of between 1000-2000 20 hours, depending upon the maximum yield being used.

\section{Present Adelphi Commercial Generators}

22 Adelphi Technology produces high intensity neutron generators based on this technology. Adelphi currently 23 makes devices with outputs of $10^{8}$ (DD-108), $10^{9}$ (DD-109) and $10^{10}$ (DD-110) fast neutrons per second (Figs. 2, 3). 24 In addition, Adelphi has built a neutron generator specialized for the production of thermal neutrons for both prompt 25 and delayed gamma neutron activation studies. The generators all have single ion beam sources and are contained in 26 stainless steel containers. The current products can either be operated continuously or pulsed. Using both a foil 27 activation technique and a neutron detector, the measured yield of the DD-109 generator is $2 \times 10^{9} \mathrm{n} / \mathrm{s}$, while the 28 maximum measured yield of the DD-108 is $1 \times 10^{8} \mathrm{n} / \mathrm{s}$. These generators are supported by a single chiller and 
standard 24" rack of power supplies and controls. The entire system is computer controlled. At the time of this

2 writing, the maximum measure yield of the DD-110 is also $2 \times 10^{9} \mathrm{n} / \mathrm{s}$, but higher yields are expected soon. The

3 original DD-110 has experienced some problems with high voltage breakdown because of its unusual rectangular

4 beam optics (e.g. $2 \mathrm{~mm} \times 30 \mathrm{~mm}$ ). The full $10^{10} \mathrm{n} / \mathrm{s}$ are expected based on the expected beam current and voltage.

5 The neutron flux depends on the ion current and acceleration voltage. The voltage is easily controlled, and the

6 current can be controlled by varying the plasma density (both by varying the excitation and gas density). This allows

7 continuous and precise control of the neutron output, and if the output is measured, a feedback loop can maintain a

8 constant output flux over extended times. Because the voltage, gas pressure and RF excitation can be computer

9 controlled, the neutron generators have been equipped with software to allow their control remotely and even over

10 an internet connection. Generators using the D-D reaction have the further advantage that the system can be opened

11 and serviced, greatly increasing their lifetime. The generator head uses a serviceable stainless steel housing with

12 conflate copper-gasket seals that can be easily removed, permitting most of the components in the generator head to

13 be easily serviced and replaced (as long as these components have not become too radioactive). All high voltage

14 and RF power are safely sealed away, permitting safe operation.

15 The compactness of the generators permits them to be safely housed in polyethylene moderators measuring 16 roughly $60 \times 60 \times 80 \mathrm{~cm}^{3}$ with additional lead and borated polyethylene. The moderators for the DD-110 and DD-

17109 are shown in Fig. 2 and 3a. The head is partially surrounded by $3 \mathrm{~mm}$ thick lead to shield the spurious x-rays

18 generated by electrons emitted back to the plasma source from the primary ion interaction at the titanium target.

19 Surrounding the polyethylene moderator is borated polyethylene to minimize any thermal neutrons and reduce the

$202.2 \mathrm{MeV}$ gamma emission from neutron capture in hydrogen. Additional ports can be machined into the

21 polyethylene for the optimal placement of materials to be irradiated or for the extraction of either thermal or fast

22 neutrons for example PGNAA. Such arrangements have been done for PGNAA by us and LBNL [4].

\section{Pulse Generation}

24 There are numerous applications that can benefit from the production of pulsed neutron sources, such as

25 differential die-away measurements of special nuclear materials and, generally, the separation of thermal and fast

26 neutron induced signals. A plasma neutron generator can be pulsed either by varying the excitation of the plasma or 27 the acceleration field. 
One means of pulsing the neutron generator is to pulse the RF power to the plasma. We have measured the pulse length using the RF plasma light output and the thermal and fast neutron output from the generator. A light

3 pipe was used to gather light from the RF chamber so it could be measured with a fast photodiode. Using a BC-

4208 fast neutron scintillator made by ELJEN Technologies and a photomultiplier, we also measured the neutron flux

5 per pulse and pulse structure. This is shown in Fig. 4, where we plotted the number of neutrons and photodiode 6 signal (plasma light) as a function of time.

Following earlier research by LBNL [3], we achieved pulse lengths from $20 \mu$ s to 1 ms with duty factors as low 9 detector we observed $6 \mu$ s pulse fall times, and with the fast neutron detector a 8- $\mu$ s fall-off time was measured. 10 Again this is shown in Fig. 4. One can also see a plasma and neutron emission ("afterglow”) after the RF pulse is 11 extinguished that last 20 to $50 \mu$ s.

12 We also found that we could operate at low pressures and still reignite the plasma at each pulse. The minimum 13 operating pressure depends on the duty cycle. The minimum pressure is around $10 \mathrm{mTorr}$ at large duty cycles, but 14 beginning below a $10-15 \%$ duty cycle the minimum pressure required to reignite the plasma dramatically increases. 15 We also demonstrated that we could maintain plasma ignition between pulses by pre-igniting the plasma with short 16 electrical pulses. We positioned a small arc electrode ("sparkplug") in the back of the RF plasma chamber. We 17 reignited the plasma with a $10 \mathrm{kV}, 10 \mu$ s pulse to the sparkplug electrode. This HV pulse was synchronized with the 18 RF plasma pulse. This technique permitted us to have longer neutron pulses $>1$ ms with duty factors $<10 \%$ while 19 still maintaining pressures below 50 mTorr inside the plasma chamber.

20 In order to shorten the fall-off further $(<1 \mu \mathrm{s})$ and eliminate the afterglow, we used a gate electrode to quench 21 the plasma meniscus quickly. This blocks the ion extraction and eliminates the neutron production between pulses 22 due to afterglow and pulse falloff. In Fig. 1 the gate electrode is shown positioned just after the plasma extraction 23 iris . The gate works as follows: The ion source is at ground potential with positive ions streaming out of the 24 extraction aperture. If the gate electrode is biased positively (+200V), it will repel the ions and prevent them from 25 entering the shroud aperture and striking the Ti target. If the gate is biased to $-2 \mathrm{kV}$ the ions are accelerated to the 26 target. We fabricated the gate electrode and placed it in an Adelphi neutron generator. The neutron counts increased 27 with increasing gate voltage. Also, a +200V was sufficient to stop the beam entirely. With the gate electrode set to $282 \mathrm{kV}$, the generator produced a neutron pulse that matched the RF pulse structure. The neutron-pulse falloff was 
$110 \mu$ in Fig. 4. When a $15-\mu$ s pulse reverse biases the gate to $+200 \mathrm{~V}$, the neutron pulse is truncated and the

2 afterglow neutrons are eliminated. This is shown in Fig. 5. The ion afterglow is eliminated and the neutron pulse

3 falloff is truncated to $<1 \mu \mathrm{s}$. The afterglow is eliminated for the duration of the $15 \mu$ s pulse.

\section{Applications}

5 The high neutron yield offered by Adelphi's plasma sources offers a larger range of laboratory research

6 opportunities, including neutron activation studies, explosives detection, detection of special nuclear materials, 7 neutron radiography, and chemical analysis.

8 Detection of Conventional Explosives: Neutron interrogation is a valid method for determining the presence of 9 concealed conventional explosives. There are several different techniques used, with effectiveness depending on 10 the particular application. Whether thermal activation (measuring prompt or delayed gammas), fast neutron excited 11 emission, or neutron radiography are used, increased flux will increase the range of detection - a critical parameter.

12 Some of these projects have benefited from Adelphi high-output source technology. The research efforts should 13 yield a viable solution for shipping containers in the next few years.

14 Special Nuclear Material (SNM) detection: Today, the detection and verification of SNM is an important challenge 15 for the world community. The prevention of terrorists' smuggling of nuclear materials across boarders will employ 16 new detection strategies. While advances in passive detection systems will enhance the probability of detecting 17 non-shielded or weakly shielded radioactive materials, the detection of shielded SNM and in particular shielded 18 HEU poses a significant challenge that is best addressed using active detection systems. These systems can be 19 utilized to inspect cargo in shipping containers at seaports, border crossings, and air transport containers, or be 20 deployed as mobile inspection systems. For the pulse-die away technique for locating SNM, pulsing with no 21 neutrons between pulses, as demonstrated here, is important for detection of the resultant delayed fission neutrons $22[5]$.

23 Chemical Industry: PGNAA and NAA are established tools for elemental analysis and can potentially provide a 24 complete picture of all contaminants in inorganic chemicals. Importantly, many applications require a higher 25 neutron flux than currently possible with economically viable (much less than $\$ 1 \mathrm{M}$ ) sources.

\section{Acknowledgements}

27 This work was supported by the US Department of Homeland Security under Contract no. HSHQDC-08-C-00022 28 and the US Department of Energy under contract no. DE-FG02-04ER86177. 


\section{Literature Cited}

2 1. J. Reijonen, N. Andresen, F. Gicquel, R. Gough, M. King, T. Kalvas, K.-N. Leung, T.-P. Lou, H. Vainionpaa,

3 A. Antolak, D. Morse, B. Doyle, G. Miller and M. A. Piestrup, "Development of Advanced Neutron/Gamma

4 Generators for Imaging and Active Interrogation Applications,” Proceedings of SPIE Vol. $6540-55$ (SPIE,

5 Orlando, FL, 2007).

6 2. J. Reijonen, F. Gicquel, S. K. Hahto, M. King. T.-P. Lou, K. N. K.-N. Leung, "D-D neutron generator 7 development at LBNL” Applied Radiation and Isotopes, 63, 757-763 (2005).

8 3. J. Reijonen, K.N. Leung, G. Jones, "RF ion source development for neutron generation and for material 9 modification”, Rev. Sci. Instrum., 73, 934 (2002).

10 4. J. Reijonen, K.-N. Leung, R. B. Firestone, J. A. English, D. L. Perry, A. Smith, F. Gicquel, M. Sun, H.

11 Koivunoro, T. -P. Lou, B. Bandong, G. Garabedian, Zs. Revay, L. Szentmiklosi and G. Molnar, "First PGAA

12 and NAA Experimental Results from a Compact High Intensity D-D Neutron Generator,” Nucl. Instrum Meth.

13 A 522, 598 (2004).

14 5. D. Slaughter, M. Accatino, A. Bernstein, J. Candy, A. Dougan, J. Hall, A. Loshak, D. Manatt, A.Meyer, B. 15 Pohl, S. Prussin, R. Walling, D. Weirup, “Detection of Special Nuclear Material in Cargo Containers Using 16 Neutron Interrogation”, Lawrence Livermore National Laboratory, UCRL-ID-155315, August 2003. 


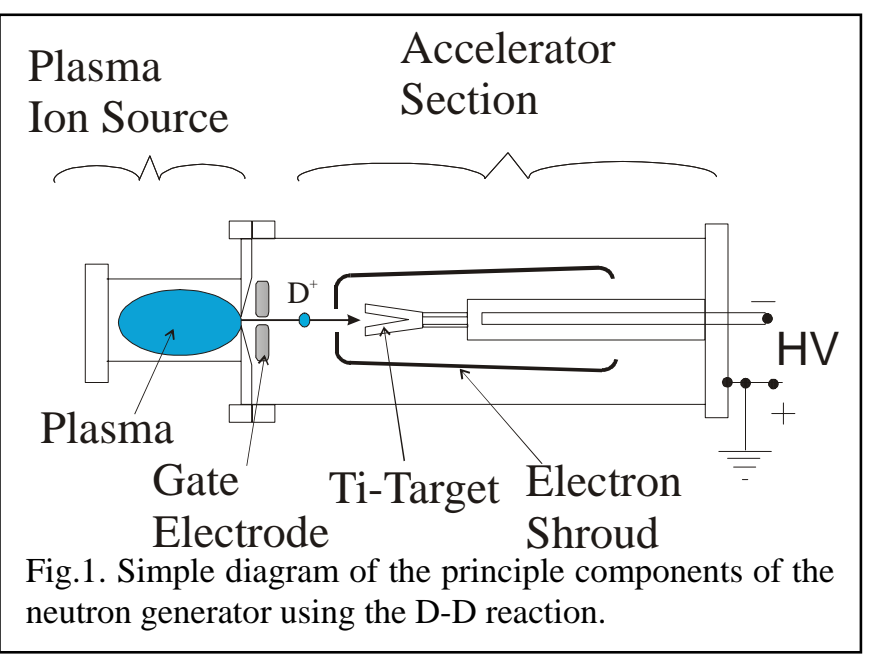




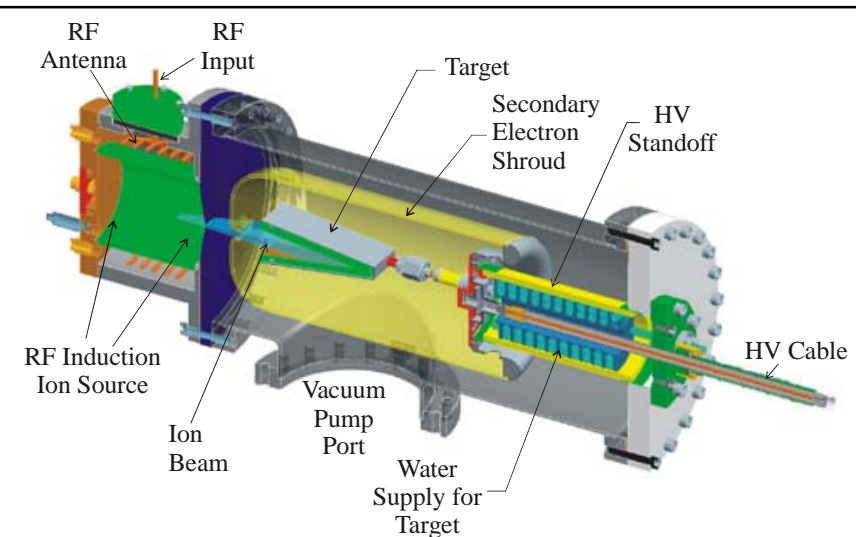

Fig. 2.a. The model DD-110 generator. This generator is expected to achieve $10^{10} \mathrm{n} / \mathrm{sec}$.

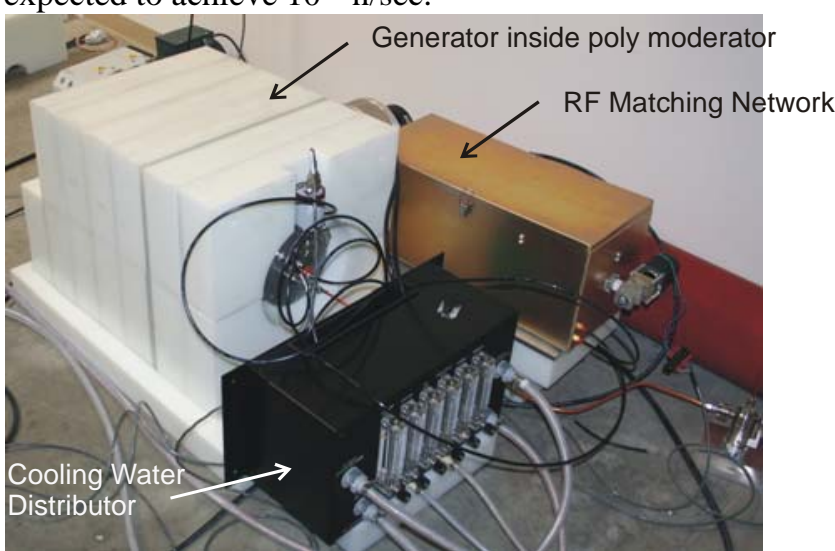

Fig. 2.b. The DD-110 surrounded by its support systems and enclosed in a polyethylene moderator. 


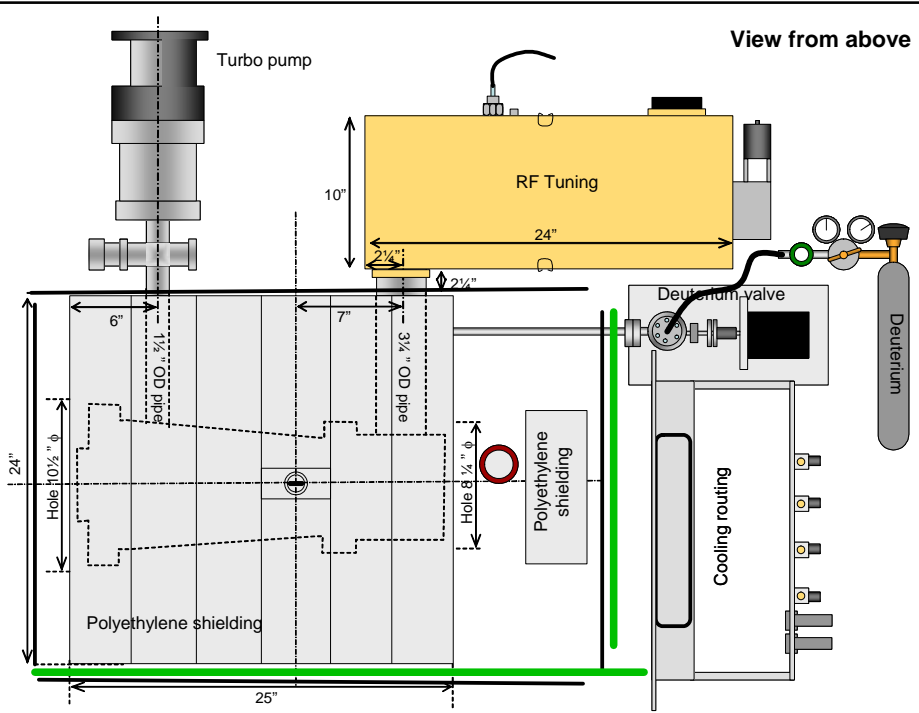

Fig. 3.a. The DD-109 enclosed in its polyethylene shield and surrounded by its support system of cooling water and deuterium gas delivery system.

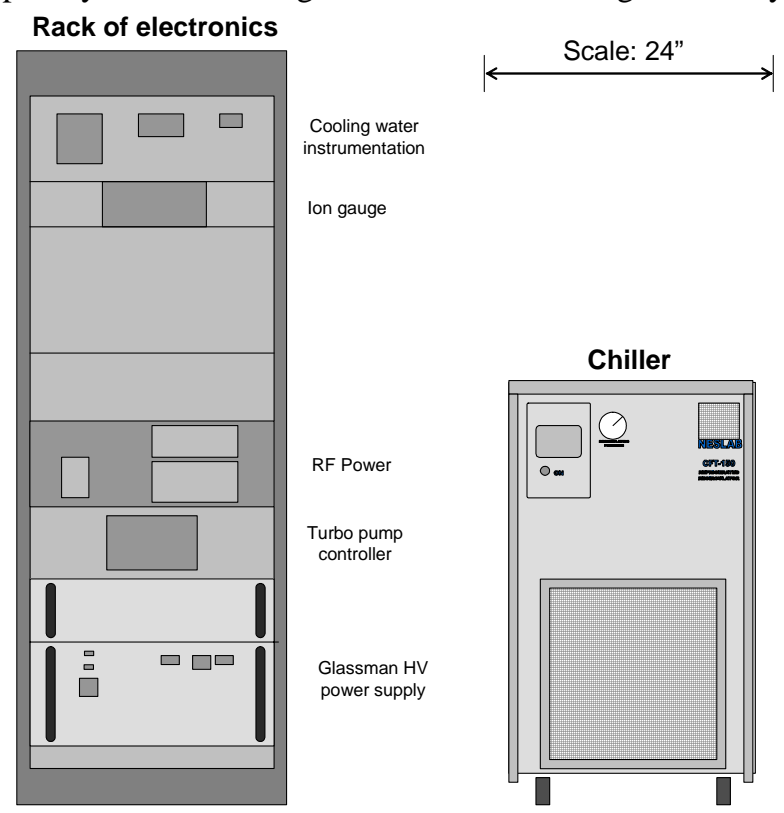

Fig. 3.b. The supporting power supply and chiller. 


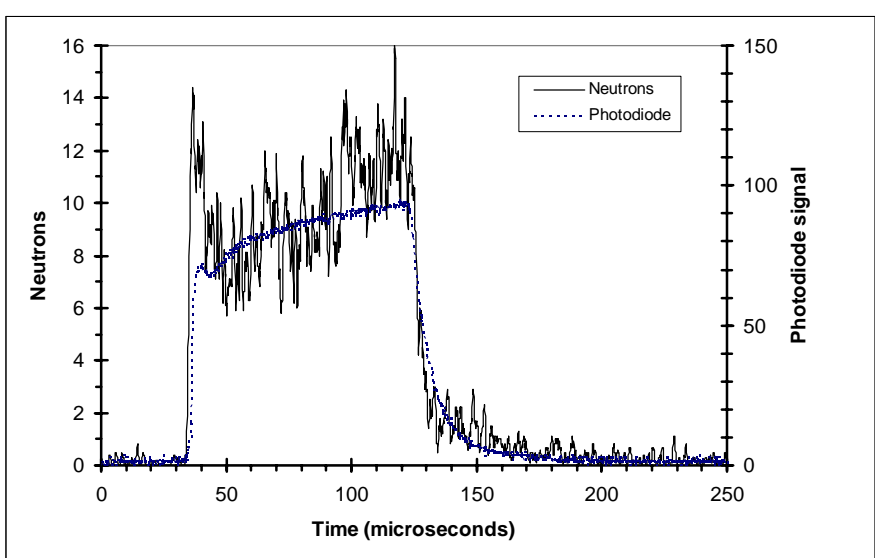

Fig.4. Measured fast neutron yield and plasma photon pulse as a function of time from the DD-108 for a $100 \mu$ s RF pulse. 


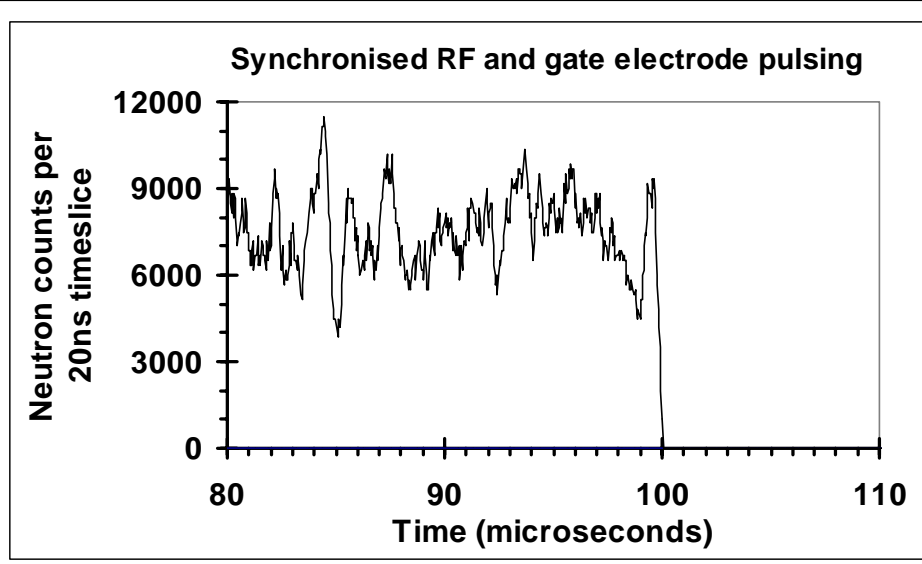

Fig. 5. The neutron count as a function of time. The gate electrode 1 was pulsed to $+200 \mathrm{~V}$ at the end of the RF pulse at $100 \mu$ s. 